\title{
Reading Wikipedia to Answer Open-Domain Questions
}

\author{
Danqi Chen* \\ Computer Science \\ Stanford University \\ Stanford, CA 94305, USA \\ danqiecs.stanford.edu
}

\author{
Adam Fisch, Jason Weston \& Antoine Bordes \\ Facebook AI Research \\ 770 Broadway \\ New York, NY 10003, USA \\ $\{$ afisch, jase, abordes $\}$ afb.com
}

\begin{abstract}
This paper proposes to tackle opendomain question answering using Wikipedia as the unique knowledge source: the answer to any factoid question is a text span in a Wikipedia article. This task of machine reading at scale combines the challenges of document retrieval (finding the relevant articles) with that of machine comprehension of text (identifying the answer spans from those articles). Our approach combines a search component based on bigram hashing and TF-IDF matching with a multi-layer recurrent neural network model trained to detect answers in Wikipedia paragraphs. Our experiments on multiple existing QA datasets indicate that (1) both modules are highly competitive with respect to existing counterparts and (2) multitask learning using distant supervision on their combination is an effective complete system on this challenging task.
\end{abstract}

\section{Introduction}

This paper considers the problem of answering factoid questions in an open-domain setting using Wikipedia as the unique knowledge source, such as one does when looking for answers in an encyclopedia. Wikipedia is a constantly evolving source of detailed information that could facilitate intelligent machines - if they are able to leverage its power. Unlike knowledge bases (KBs) such as Freebase (Bollacker et al., 2008) or DBPedia (Auer et al., 2007), which are easier for computers to process but too sparsely populated for open-domain question answering (Miller et al.,

${ }^{*}$ Most of this work was done while DC was with Facebook AI Research.
2016), Wikipedia contains up-to-date knowledge that humans are interested in. It is designed, however, for humans - not machines - to read.

Using Wikipedia articles as the knowledge source causes the task of question answering (QA) to combine the challenges of both large-scale open-domain QA and of machine comprehension of text. In order to answer any question, one must first retrieve the few relevant articles among more than 5 million items, and then scan them carefully to identify the answer. We term this setting, machine reading at scale (MRS). Our work treats Wikipedia as a collection of articles and does not rely on its internal graph structure. As a result, our approach is generic and could be switched to other collections of documents, books, or even daily updated newspapers.

Large-scale QA systems like IBM's DeepQA (Ferrucci et al., 2010) rely on multiple sources to answer: besides Wikipedia, it is also paired with KBs, dictionaries, and even news articles, books, etc. As a result, such systems heavily rely on information redundancy among the sources to answer correctly. Having a single knowledge source forces the model to be very precise while searching for an answer as the evidence might appear only once. This challenge thus encourages research in the ability of a machine to read, a key motivation for the machine comprehension subfield and the creation of datasets such as SQuAD (Rajpurkar et al., 2016), CNN/Daily Mail (Hermann et al., 2015) and CBT (Hill et al., 2016).

However, those machine comprehension resources typically assume that a short piece of relevant text is already identified and given to the model, which is not realistic for building an opendomain QA system. In sharp contrast, methods that use KBs or information retrieval over documents have to employ search as an integral part of 
the solution. Instead MRS is focused on simultaneously maintaining the challenge of machine comprehension, which requires the deep understanding of text, while keeping the realistic constraint of searching over a large open resource.

In this paper, we show how multiple existing QA datasets can be used to evaluate MRS by requiring an open-domain system to perform well on all of them at once. We develop DrQA, a strong system for question answering from Wikipedia composed of: (1) Document Retriever, a module using bigram hashing and TF-IDF matching designed to, given a question, efficiently return a subset of relevant articles and (2) Document Reader, a multi-layer recurrent neural network machine comprehension model trained to detect answer spans in those few returned documents. Figure 1 gives an illustration of DrQA.

Our experiments show that Document Retriever outperforms the built-in Wikipedia search engine and that Document Reader reaches state-of-theart results on the very competitive SQuAD benchmark (Rajpurkar et al., 2016). Finally, our full system is evaluated using multiple benchmarks. In particular, we show that performance is improved across all datasets through the use of multitask learning and distant supervision compared to single task training.

\section{Related Work}

Open-domain QA was originally defined as finding answers in collections of unstructured documents, following the setting of the annual TREC competitions $^{1}$. With the development of KBs, many recent innovations have occurred in the context of QA from KBs with the creation of resources like WebQuestions (Berant et al., 2013) and SimpleQuestions (Bordes et al., 2015) based on the Freebase KB (Bollacker et al., 2008), or on automatically extracted KBs, e.g., OpenIE triples and NELL (Fader et al., 2014). However, KBs have inherent limitations (incompleteness, fixed schemas) that motivated researchers to return to the original setting of answering from raw text.

A second motivation to cast a fresh look at this problem is that of machine comprehension of text, i.e., answering questions after reading a short text or story. That subfield has made considerable progress recently thanks to new deep learning architectures like attention-based and memory-

\footnotetext{
${ }^{1}$ http://trec.nist.gov/data/qamain.html
}

augmented neural networks (Bahdanau et al., 2015; Weston et al., 2015; Graves et al., 2014) and release of new training and evaluation datasets like QuizBowl (Iyyer et al., 2014), CNN/Daily Mail based on news articles (Hermann et al., 2015), CBT based on children books (Hill et al., 2016), or SQuAD (Rajpurkar et al., 2016) and WikiReading (Hewlett et al., 2016), both based on Wikipedia. An objective of this paper is to test how such new methods can perform in an open-domain QA framework.

QA using Wikipedia as a resource has been explored previously. Ryu et al. (2014) perform opendomain QA using a Wikipedia-based knowledge model. They combine article content with multiple other answer matching modules based on different types of semi-structured knowledge such as infoboxes, article structure, category structure, and definitions. Similarly, Ahn et al. (2004) also combine Wikipedia as a text resource with other resources, in this case with information retrieval over other documents. Buscaldi and Rosso (2006) also mine knowledge from Wikipedia for QA. Instead of using it as a resource for seeking answers to questions, they focus on validating answers returned by their QA system, and use Wikipedia categories for determining a set of patterns that should fit with the expected answer. In our work, we consider the comprehension of text only, and use Wikipedia text documents as the sole resource in order to emphasize the task of machine reading at scale, as described in the introduction.

There are a number of highly developed full pipeline QA approaches using either the Web, as does QuASE (Sun et al., 2015), or Wikipedia as a resource, as do Microsoft's AskMSR (Brill et al., 2002), IBM's DeepQA (Ferrucci et al., 2010) and YodaQA (Baudiš, 2015; Baudiš and Šedivỳ, 2015) - the latter of which is open source and hence reproducible for comparison purposes. AskMSR is a search-engine based QA system that relies on "data redundancy rather than sophisticated linguistic analyses of either questions or candidate answers", i.e., it does not focus on machine comprehension, as we do. DeepQA is a very sophisticated system that relies on both unstructured information including text documents as well as structured data such as KBs, databases and ontologies to generate candidate answers or vote over evidence. YodaQA is an open source system modeled after DeepQA, similarly combining websites, 


\section{Open-domain QA}

SQuAD, TREC, WebQuestions, WikiMovies

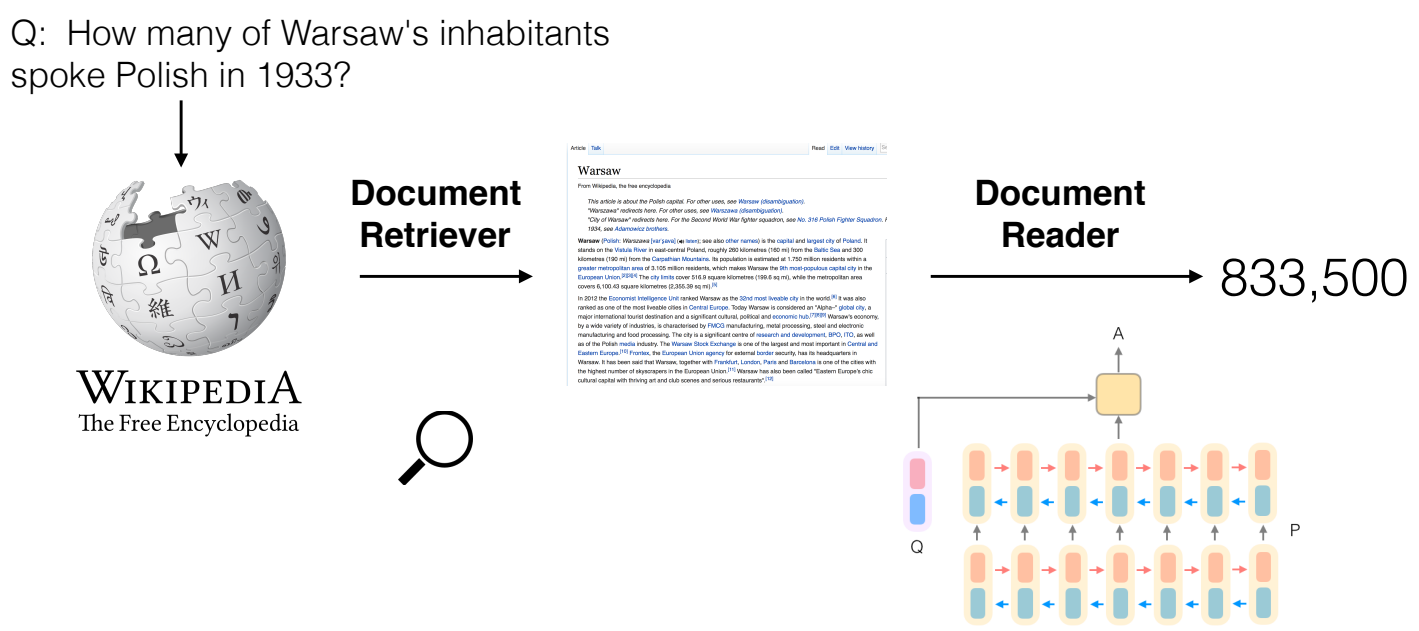

Figure 1: An overview of our question answering system DrQA.

information extraction, databases and Wikipedia in particular. Our comprehension task is made more challenging by only using a single resource. Comparing against these methods provides a useful datapoint for an "upper bound" benchmark on performance.

Multitask learning (Caruana, 1998) and task transfer have a rich history in machine learning (e.g., using ImageNet in the computer vision community (Huh et al., 2016)), as well as in NLP in particular (Collobert and Weston, 2008). Several works have attempted to combine multiple QA training datasets via multitask learning to (i) achieve improvement across the datasets via task transfer; and (ii) provide a single general system capable of asking different kinds of questions due to the inevitably different data distributions across the source datasets. Fader et al. (2014) used WebQuestions, TREC and WikiAnswers with four KBs as knowledge sources and reported improvement on the latter two datasets through multitask learning. Bordes et al. (2015) combined WebQuestions and SimpleQuestions using distant supervision with Freebase as the KB to give slight improvements on both datasets, although poor performance was reported when training on only one dataset and testing on the other, showing that task transfer is indeed a challenging subject; see also (Kadlec et al., 2016) for a similar conclusion. Our work follows similar themes, but in the setting of having to retrieve and then read text documents, rather than using a $\mathrm{KB}$, with positive results.

\section{Our System: DrQA}

In the following we describe our system DrQA for MRS which consists of two components: (1) the Document Retriever module for finding relevant articles and (2) a machine comprehension model, Document Reader, for extracting answers from a single document or a small collection of documents.

\subsection{Document Retriever}

Following classical QA systems, we use an efficient (non-machine learning) document retrieval system to first narrow our search space and focus on reading only articles that are likely to be relevant. A simple inverted index lookup followed by term vector model scoring performs quite well on this task for many question types, compared to the built-in ElasticSearch based Wikipedia Search API (Gormley and Tong, 2015). Articles and questions are compared as TF-IDF weighted bag-ofword vectors. We further improve our system by taking local word order into account with n-gram features. Our best performing system uses bigram counts while preserving speed and memory efficiency by using the hashing of (Weinberger et al., 2009) to map the bigrams to $2^{24}$ bins with an unsigned murmur 3 hash.

We use Document Retriever as the first part of our full model, by setting it to return 5 Wikipedia 
articles given any question. Those articles are then processed by Document Reader.

\subsection{Document Reader}

Our Document Reader model is inspired by the recent success of neural network models on machine comprehension tasks, in a similar spirit to the AttentiveReader described in (Hermann et al., 2015; Chen et al., 2016).

Given a question $q$ consisting of $l$ tokens $\left\{q_{1}, \ldots, q_{l}\right\}$ and a document or a small set of documents of $n$ paragraphs where a single paragraph $p$ consists of $m$ tokens $\left\{p_{1}, \ldots, p_{m}\right\}$, we develop an RNN model that we apply to each paragraph in turn and then finally aggregate the predicted answers. Our method works as follows:

Paragraph encoding We first represent all tokens $p_{i}$ in a paragraph $p$ as a sequence of feature vectors $\tilde{\mathbf{p}}_{i} \in \mathbb{R}^{d}$ and pass them as the input to a recurrent neural network and thus obtain:

$$
\left\{\mathbf{p}_{1}, \ldots, \mathbf{p}_{m}\right\}=\operatorname{RNN}\left(\left\{\tilde{\mathbf{p}}_{1}, \ldots, \tilde{\mathbf{p}}_{m}\right\}\right),
$$

where $\mathbf{p}_{i}$ is expected to encode useful context information around token $p_{i}$. Specifically, we choose to use a multi-layer bidirectional long short-term memory network (LSTM), and take $\mathbf{p}_{i}$ as the concatenation of each layer's hidden units in the end.

The feature vector $\tilde{\mathbf{p}}_{i}$ is comprised of the following parts:

- Word embeddings: $f_{\text {emb }}\left(p_{i}\right)=\mathbf{E}\left(p_{i}\right)$. We use the 300-dimensional Glove word embeddings trained from $840 \mathrm{~B}$ Web crawl data (Pennington et al., 2014). We keep most of the pre-trained word embeddings fixed and only fine-tune the 1000 most frequent question words because the representations of some key words such as what, how, which, many could be crucial for QA systems.

- Exact match: $f_{\text {exact_match }}\left(p_{i}\right)=\mathbb{I}\left(p_{i} \in q\right)$. We use three simple binary features, indicating whether $p_{i}$ can be exactly matched to one question word in $q$, either in its original, lowercase or lemma form. These simple features turn out to be extremely helpful, as we will show in Section 5.

- Token features: $f_{\text {token }}\left(p_{i}\right)=\left(\operatorname{POS}\left(p_{i}\right), \operatorname{NER}\left(p_{i}\right), \operatorname{TF}\left(p_{i}\right)\right)$.
We also add a few manual features which reflect some properties of token $p_{i}$ in its context, which include its part-of-speech (POS) and named entity recognition (NER) tags and its (normalized) term frequency (TF).

\section{- Aligned question embedding:}

Following (Lee et al., 2016) and other recent works, the last part we incorporate is an aligned question embedding $f_{\text {align }}\left(p_{i}\right)=$ $\sum_{j} a_{i, j} \mathbf{E}\left(q_{j}\right)$, where the attention score $a_{i, j}$ captures the similarity between $p_{i}$ and each question words $q_{j}$. Specifically, $a_{i, j}$ is computed by the dot products between nonlinear mappings of word embeddings:

$$
a_{i, j}=\frac{\exp \left(\alpha\left(\mathbf{E}\left(p_{i}\right)\right) \cdot \alpha\left(\mathbf{E}\left(q_{j}\right)\right)\right)}{\sum_{j^{\prime}} \exp \left(\alpha\left(\mathbf{E}\left(p_{i}\right)\right) \cdot \alpha\left(\mathbf{E}\left(q_{j^{\prime}}\right)\right)\right)},
$$

and $\alpha(\cdot)$ is a single dense layer with ReLU nonlinearity. Compared to the exact match features, these features add soft alignments between similar but non-identical words (e.g., car and vehicle).

Question encoding The question encoding is simpler, as we only apply another recurrent neural network on top of the word embeddings of $q_{i}$ and combine the resulting hidden units into one single vector: $\left\{\mathbf{q}_{1}, \ldots, \mathbf{q}_{l}\right\} \rightarrow \mathbf{q}$. We compute $\mathbf{q}=\sum_{j} b_{j} \mathbf{q}_{j}$ where $b_{j}$ encodes the importance of each question word:

$$
b_{j}=\frac{\exp \left(\mathbf{w} \cdot \mathbf{q}_{j}\right)}{\sum_{j^{\prime}} \exp \left(\mathbf{w} \cdot \mathbf{q}_{j^{\prime}}\right)},
$$

and $\mathbf{w}$ is a weight vector to learn.

Prediction At the paragraph level, the goal is to predict the span of tokens that is most likely the correct answer. We take the the paragraph vectors $\left\{\mathbf{p}_{1}, \ldots, \mathbf{p}_{m}\right\}$ and the question vector $\mathbf{q}$ as input, and simply train two classifiers independently for predicting the two ends of the span. Concretely, we use a bilinear term to capture the similarity between $\mathbf{p}_{i}$ and $\mathbf{q}$ and compute the probabilities of each token being start and end as:

$$
\begin{aligned}
P_{\text {start }}(i) & \propto \exp \left(\mathbf{p}_{i} \mathbf{W}_{s} \mathbf{q}\right) \\
P_{\text {end }}(i) & \propto \exp \left(\mathbf{p}_{i} \mathbf{W}_{e} \mathbf{q}\right)
\end{aligned}
$$

During prediction, we choose the best span from token $i$ to token $i^{\prime}$ such that $i \leq i^{\prime} \leq i+15$ and $P_{\text {start }}(i) \times P_{\text {end }}\left(i^{\prime}\right)$ is maximized. To make scores 
compatible across paragraphs in one or several retrieved documents, we use the unnormalized exponential and take argmax over all considered paragraph spans for our final prediction.

\section{Data}

Our work relies on three types of data: (1) Wikipedia that serves as our knowledge source for finding answers, (2) the SQuAD dataset which is our main resource to train Document Reader and (3) three more QA datasets (CuratedTREC, WebQuestions and WikiMovies) that in addition to SQuAD, are used to test the open-domain QA abilities of our full system, and to evaluate the ability of our model to learn from multitask learning and distant supervision. Statistics of the datasets are given in Table 2.

\subsection{Wikipedia (Knowledge Source)}

We use the 2016-12-21 dump ${ }^{2}$ of English Wikipedia for all of our full-scale experiments as the knowledge source used to answer questions. For each page, only the plain text is extracted and all structured data sections such as lists and figures are stripped. ${ }^{3}$ After discarding internal disambiguation, list, index, and outline pages, we retain 5,075,182 articles consisting of 9,008,962 unique uncased token types.

\subsection{SQuAD}

The Stanford Question Answering Dataset (SQuAD) (Rajpurkar et al., 2016) is a dataset for machine comprehension based on Wikipedia. The dataset contains $87 \mathrm{k}$ examples for training and $10 \mathrm{k}$ for development, with a large hidden test set which can only be accessed by the SQuAD creators. Each example is composed of a paragraph extracted from a Wikipedia article and an associated human-generated question. The answer is always a span from this paragraph and a model is given credit if its predicted answer matches it. Two evaluation metrics are used: exact string match (EM) and F1 score, which measures the weighted average of precision and recall at the token level.

In the following, we use $\mathrm{SQuAD}$ for training and evaluating our Document Reader for the standard machine comprehension task given the rel-

\footnotetext{
${ }^{2}$ https://dumps.wikimedia.org/enwiki/ latest

${ }^{3}$ We use the WikiExtractor script: https://github. com/attardi/wikiextractor.
}

evant paragraph as defined in (Rajpurkar et al., 2016). For the task of evaluating open-domain question answering over Wikipedia, we use the SQuAD development set QA pairs only, and we ask systems to uncover the correct answer spans without having access to the associated paragraphs. That is, a model is required to answer a question given the whole of Wikipedia as a resource; it is not given the relevant paragraph as in the standard SQuAD setting.

\subsection{Open-domain QA Evaluation Resources}

SQuAD is one of the largest general purpose QA datasets currently available. SQuAD questions have been collected via a process involving showing a paragraph to each human annotator and asking them to write a question. As a result, their distribution is quite specific. We hence propose to train and evaluate our system on other datasets developed for open-domain QA that have been constructed in different ways (not necessarily in the context of answering from Wikipedia).

CuratedTREC This dataset is based on the benchmarks from the TREC QA tasks that have been curated by Baudiš and Šedivỳ (2015). We use the large version, which contains a total of 2,180 questions extracted from the datasets from TREC 1999, 2000, 2001 and 2002. ${ }^{4}$

WebQuestions Introduced in (Berant et al., 2013), this dataset is built to answer questions from the Freebase KB. It was created by crawling questions through the Google Suggest API, and then obtaining answers using Amazon Mechanical Turk. We convert each answer to text by using entity names so that the dataset does not reference Freebase IDs and is purely made of plain text question-answer pairs.

WikiMovies This dataset, introduced in (Miller et al., 2016), contains 96k question-answer pairs in the domain of movies. Originally created from the $\mathrm{OMDb}$ and MovieLens databases, the examples are built such that they can also be answered by using a subset of Wikipedia as the knowledge source (the title and the first section of articles from the movie domain).

\footnotetext{
${ }^{4}$ This dataset is available at https://github.com/ brmson/dataset-factoid-curated.
} 


\begin{tabular}{|c|c|c|}
\hline Dataset & Example & Article / Paragraph \\
\hline SQuAD & $\begin{array}{l}\text { Q: How many provinces did the Ottoman } \\
\text { empire contain in the } 17 \text { th century? } \\
\text { A: } 32\end{array}$ & $\begin{array}{l}\text { Article: Ottoman Empire } \\
\text { Paragraph: ... At the beginning of the } 17 \text { th century the em- } \\
\text { pire contained } 32 \text { provinces and numerous vassal states. Some } \\
\text { of these were later absorbed into the Ottoman Empire, while } \\
\text { others were granted various types of autonomy during the } \\
\text { course of centuries. }\end{array}$ \\
\hline CuratedTREC & $\begin{array}{l}\text { Q: What U.S. state's motto is "Live free } \\
\text { or Die"? } \\
\text { A: New Hampshire }\end{array}$ & $\begin{array}{l}\text { Article: Live Free or Die } \\
\text { Paragraph: "Live Free or Die" is the official motto of the } \\
\text { U.S. state of New Hampshire, adopted by the state in } 1945 \text {. It } \\
\text { is possibly the best-known of all state mottos, partly because it } \\
\text { conveys an assertive independence historically found in Amer- } \\
\text { ican political philosophy and partly because of its contrast to } \\
\text { the milder sentiments found in other state mottos. }\end{array}$ \\
\hline WebQuestions & $\begin{array}{l}\text { Q: What part of the atom did Chadwick } \\
\text { discover? } \\
\text { A: neutron }\end{array}$ & $\begin{array}{l}\text { Article: Atom } \\
\text { Paragraph: ... The atomic mass of these isotopes varied by } \\
\text { integer amounts, called the whole number rule. The explana- } \\
\text { tion for these different isotopes awaited the discovery of the } \\
\text { neutron, an uncharged particle with a mass similar to the pro- } \\
\text { ton, by the physicist James Chadwick in } 1932 . . .\end{array}$ \\
\hline WikiMovies & $\begin{array}{l}\text { Q: Who wrote the film Gigli? } \\
\text { A: Martin Brest }\end{array}$ & $\begin{array}{l}\text { Article: Gigli } \\
\text { Paragraph: Gigli is a } 2003 \text { American romantic comedy film } \\
\text { written and directed by Martin Brest and starring Ben Affleck, } \\
\text { Jennifer Lopez, Justin Bartha, Al Pacino, Christopher Walken, } \\
\text { and Lainie Kazan. }\end{array}$ \\
\hline
\end{tabular}

Table 1: Example training data from each QA dataset. In each case we show an associated paragraph where distant supervision (DS) correctly identified the answer within it, which is highlighted.

\begin{tabular}{|c|c|c|c|}
\hline \multirow[t]{2}{*}{ Dataset } & \multicolumn{2}{|c|}{ Train } & Test \\
\hline & Plain & DS & \\
\hline SQuAD & 87,599 & 71,231 & $10,570^{\dagger}$ \\
\hline CuratedTREC & $1,486^{*}$ & 3,464 & 694 \\
\hline WebQuestions & $3,778^{*}$ & 4,602 & 2,032 \\
\hline WikiMovies & $96,185^{*}$ & 36,301 & 9,952 \\
\hline
\end{tabular}

Table 2: Number of questions for each dataset used in this paper. DS: distantly supervised training data. *: These training sets are not used as is because no paragraph is associated with each question. ${ }^{\dagger}$ : Corresponds to SQuAD development set.

\subsection{Distantly Supervised Data}

All the QA datasets presented above contain training portions, but CuratedTREC, WebQuestions and WikiMovies only contain question-answer pairs, and not an associated document or paragraph as in SQuAD, and hence cannot be used for training Document Reader directly. Following previous work on distant supervision (DS) for relation extraction (Mintz et al., 2009), we use a procedure to automatically associate paragraphs to such training examples, and then add these examples to our training set.

We use the following process for each questionanswer pair to build our training set. First, we

\begin{tabular}{l|c|cc}
\hline Dataset & Wiki & \multicolumn{2}{c}{ Doc. Retriever } \\
& Search & plain & +bigrams \\
\hline SQuAD & 62.7 & 76.1 & $\mathbf{7 7 . 8}$ \\
CuratedTREC & 81.0 & 85.2 & $\mathbf{8 6 . 0}$ \\
WebQuestions & 73.7 & $\mathbf{7 5 . 5}$ & 74.4 \\
WikiMovies & 61.7 & 54.4 & $\mathbf{7 0 . 3}$ \\
\hline
\end{tabular}

Table 3: Document retrieval results. \% of questions for which the answer segment appears in one of the top 5 pages returned by the method.

run Document Retriever on the question to retrieve the top 5 Wikipedia articles. All paragraphs from those articles without an exact match of the known answer are directly discarded. All paragraphs shorter than 25 or longer than 1500 characters are also filtered out. If any named entities are detected in the question, we remove any paragraph that does not contain them at all. For every remaining paragraph in each retrieved page, we score all positions that match an answer using unigram and bigram overlap between the question and a 20 token window, keeping up to the top 5 paragraphs with the highest overlaps. If there is no paragraph with non-zero overlap, the example is discarded; otherwise we add each found pair to our DS training dataset. Some examples are shown in Table 1 and data statistics are given in Table 2. 
Note that we can also generate additional DS data for SQuAD by trying to find mentions of the answers not just in the paragraph provided, but also from other pages or the same page that the given paragraph was in. We observe that around half of the DS examples come from pages outside of the articles used in SQuAD.

\section{Experiments}

This section first presents evaluations of our Document Retriever and Document Reader modules separately, and then describes tests of their combination, DrQA, for open-domain QA on the full Wikipedia.

\subsection{Finding Relevant Articles}

We first examine the performance of our Document Retriever module on all the QA datasets. Table 3 compares the performance of the two approaches described in Section 3.1 with that of the Wikipedia Search Engine ${ }^{5}$ for the task of finding articles that contain the answer given a question. Specifically, we compute the ratio of questions for which the text span of any of their associated answers appear in at least one the top 5 relevant pages returned by each system. Results on all datasets indicate that our simple approach outperforms Wikipedia Search, especially with bigram hashing. We also compare doing retrieval with Okapi BM25 or by using cosine distance in the word embeddings space (by encoding questions and articles as bag-of-embeddings), both of which we find performed worse.

\subsection{Reader Evaluation on SQuAD}

Next we evaluate our Document Reader component on the standard SQuAD evaluation (Rajpurkar et al., 2016).

Implementation details We use 3-layer bidirectional LSTMs with $h=128$ hidden units for both paragraph and question encoding. We apply the Stanford CoreNLP toolkit (Manning et al., 2014) for tokenization and also generating lemma, partof-speech, and named entity tags.

Lastly, all the training examples are sorted by the length of paragraph and divided into minibatches of 32 examples each. We use Adamax for optimization as described in (Kingma and Ba,

\footnotetext{
${ }^{5}$ We use the Wikipedia Search API https://www. mediawiki.org/wiki/API : Search.
}

2014). Dropout with $p=0.3$ is applied to word embeddings and all the hidden units of LSTMs.

Result and analysis Table 4 presents our evaluation results on both development and test sets. SQuAD has been a very competitive machine comprehension benchmark since its creation and we only list the best-performing systems in the table. Our system (single model) can achieve 70.0\% exact match and $79.0 \%$ F1 scores on the test set, which surpasses all the published results and can match the top performance on the SQuAD leaderboard at the time of writing. Additionally, we think that our model is conceptually simpler than most of the existing systems. We conducted an ablation analysis on the feature vector of paragraph tokens. As shown in Table 5 all the features contribute to the performance of our final system. Without the aligned question embedding feature (only word embedding and a few manual features), our system is still able to achieve $\mathrm{F} 1$ over $77 \%$. More interestingly, if we remove both $f_{\text {aligned }}$ and $f_{\text {exact_match }}$, the performance drops dramatically, so we conclude that both features play a similar but complementary role in the feature representation related to the paraphrased nature of a question vs. the context around an answer.

\subsection{Full Wikipedia Question Answering}

Finally, we assess the performance of our full system DrQA for answering open-domain questions using the four datasets introduced in Section 4. We compare three versions of DrQA which evaluate the impact of using distant supervision and multitask learning across the training sources provided to Document Reader (Document Retriever remains the same for each case):

- SQuAD: A single Document Reader model is trained on the SQuAD training set only and used on all evaluation sets.

- Fine-tune (DS): A Document Reader model is pre-trained on SQuAD and then fine-tuned for each dataset independently using its distant supervision (DS) training set.

- Multitask (DS): A single Document Reader model is jointly trained on the SQuAD training set and all the DS sources.

For the full Wikipedia setting we use a streamlined model that does not use the CoreNLP parsed $f_{\text {token }}$ features or lemmas for $f_{\text {exact_match }}$. We 


\begin{tabular}{|c|c|c|c|c|}
\hline \multirow[t]{2}{*}{ Method } & \multicolumn{2}{|c|}{ Dev } & \multicolumn{2}{|c|}{ Test } \\
\hline & EM & F1 & EM & F1 \\
\hline Dynamic Coattention Networks (Xiong et al., 2016) & 65.4 & 75.6 & 66.2 & 75.9 \\
\hline Multi-Perspective Matching (Wang et al., 2016) & 66.1 & 75.8 & 65.5 & 75.1 \\
\hline BiDAF (Seo et al., 2016) & 67.7 & 77.3 & 68.0 & 77.3 \\
\hline R-net ${ }^{\dagger}$ & $\mathrm{n} / \mathrm{a}$ & $\mathrm{n} / \mathrm{a}$ & 71.3 & 79.7 \\
\hline DrQA (Our model, Document Reader Only) & 69.5 & 78.8 & 70.0 & 79.0 \\
\hline
\end{tabular}

Table 4: Evaluation results on the SQuAD dataset (single model only). ${ }^{\dagger}:$ Test results reflect the SQuAD leaderboard (https://stanford-qa.com) as of Feb 6, 2017.

\begin{tabular}{l|l}
\hline Features & F1 \\
\hline Full & 78.8 \\
\hline No $f_{\text {token }}$ & $78.0(-0.8)$ \\
No $f_{\text {exact_match }}$ & $77.3(-1.5)$ \\
No $f_{\text {aligned }}$ & $77.3(-1.5)$ \\
No $f_{\text {aligned }}$ and $f_{\text {exact_match }}$ & $59.4(-19.4)$ \\
\hline
\end{tabular}

Table 5: Feature ablation analysis of the paragraph representations of our Document Reader. Results are reported on the SQuAD development set.

find that while these help for more exact paragraph reading in SQuAD, they don't improve results in the full setting. Additionally, WebQuestions and WikiMovies provide a list of candidate answers (e.g., 1.6 million Freebase entity strings for WebQuestions) and we restrict the answer span must be in this list during prediction.

Results Table 6 presents the results. Despite the difficulty of the task compared to machine comprehension (where you are given the right paragraph) and unconstrained QA (using redundant resources), DrQA still provides reasonable performance across all four datasets.

We are interested in a single, full system that can answer any question using Wikipedia. The single model trained only on SQuAD is outperformed on all four of the datasets by the multitask model that uses distant supervision. However performance when training on SQuAD alone is not far behind, indicating that task transfer is occurring. The majority of the improvement from SQuAD to Multitask (DS) however is likely not from task transfer as fine-tuning on each dataset alone using DS also gives improvements, showing that is is the introduction of extra data in the same domain that helps. Nevertheless, the best single model that we can find is our overall goal, and that is the Multitask (DS) system.
We compare to an unconstrained QA system using redundant resources (not just Wikipedia), YodaQA (Baudiš, 2015), giving results which were previously reported on CuratedTREC and WebQuestions. Despite the increased difficulty of our task, it is reassuring that our performance is not too far behind on CuratedTREC (31.3 vs. 25.4). The gap is slightly bigger on WebQuestions, likely because this dataset was created from the specific structure of Freebase which YodaQA uses directly.

DrQA's performance on SQuAD compared to its Document Reader component on machine comprehension in Table 4 shows a large drop (from 69.5 to 27.1 ) as we now are given Wikipedia to read, not a single paragraph. Given the correct document (but not the paragraph) we can achieve 49.4, indicating many false positives come from highly topical sentences. This is despite the fact that the Document Retriever works relatively well (77.8\% of the time retrieving the answer, see Table 3). It is worth noting that a large part of the drop comes from the nature of the SQuAD questions. They were written with a specific paragraph in mind, thus their language can be ambiguous when the context is removed. Additional resources other than SQuAD, specifically designed for MRS, might be needed to go further.

\section{Conclusion}

We studied the task of machine reading at scale, by using Wikipedia as the unique knowledge source for open-domain QA. Our results indicate that MRS is a key challenging task for researchers to focus on. Machine comprehension systems alone cannot solve the overall task. Our method integrates search, distant supervision, and multitask learning to provide an effective complete system. Evaluating the individual components as well as the full system across multiple benchmarks showed the efficacy of our approach. 


\begin{tabular}{l|c|ccc}
\hline Dataset & YodaQA & \multicolumn{3}{|c}{ DrQA } \\
& & SQuAD & +Fine-tune (DS) & +Multitask (DS) \\
\hline SQuAD (All Wikipedia) & $\mathrm{n} / \mathrm{a}$ & 27.1 & 28.4 & 29.8 \\
CuratedTREC & 31.3 & 19.7 & 25.7 & 25.4 \\
WebQuestions & 39.8 & 11.8 & 19.5 & 20.7 \\
WikiMovies & $\mathrm{n} / \mathrm{a}$ & 24.5 & 34.3 & 36.5 \\
\hline
\end{tabular}

Table 6: Full Wikipedia results. Top-1 exact-match accuracy (in \%, using SQuAD eval script). +Finetune (DS): Document Reader models trained on SQuAD and fine-tuned on each DS training set independently. +Multitask (DS): Document Reader single model trained on SQuAD and all the distant supervision (DS) training sets jointly. YodaQA results are extracted from https://github.com/brmson/ yodaqa/wiki/Benchmarks and use additional resources such as Freebase and DBpedia, see Section 2.

Future work should aim to improve over our DrQA system. Two obvious angles of attack are: (i) incorporate the fact that Document Reader aggregates over multiple paragraphs and documents directly in the training, as it currently trains on paragraphs independently; and (ii) perform endto-end training across the Document Retriever and Document Reader pipeline, rather than independent systems.

\section{Acknowledgments}

The authors thank Pranav Rajpurkar for testing Document Reader on the test set of SQuAD.

\section{References}

David Ahn, Valentin Jijkoun, Gilad Mishne, Karin Mller, Maarten de Rijke, and Stefan Schlobach. 2004. Using wikipedia at the trec qa track. In Proceedings of TREC 2004.

Sören Auer, Christian Bizer, Georgi Kobilarov, Jens Lehmann, Richard Cyganiak, and Zachary Ives. 2007. Dbpedia: A nucleus for a web of open data. In The semantic web, Springer, pages 722-735.

Dzmitry Bahdanau, Kyunghyun Cho, and Yoshua Bengio. 2015. Neural machine translation by jointly learning to align and translate. In International Conference on Learning Representations (ICLR).

Petr Baudiš. 2015. YodaQA: a modular question answering system pipeline. In POSTER 2015-19th International Student Conference on Electrical Engineering. pages 1156-1165.

Petr Baudiš and Jan Šedivỳ. 2015. Modeling of the question answering task in the YodaQA system. In International Conference of the CrossLanguage Evaluation Forum for European Languages. Springer, pages 222-228.

Jonathan Berant, Andrew Chou, Roy Frostig, and Percy Liang. 2013. Semantic parsing on freebase from question-answer pairs. In Empirical Methods in Natural Language Processing (EMNLP). pages 1533-1544.

Kurt Bollacker, Colin Evans, Praveen Paritosh, Tim Sturge, and Jamie Taylor. 2008. Freebase: a collaboratively created graph database for structuring human knowledge. In Proceedings of the 2008 ACM SIGMOD international conference on Management of data. AcM, pages 1247-1250.

Antoine Bordes, Nicolas Usunier, Sumit Chopra, and Jason Weston. 2015. Large-scale simple question answering with memory networks. arXiv preprint arXiv:1506.02075

Eric Brill, Susan Dumais, and Michele Banko. 2002. An analysis of the AskMSR question-answering system. In Empirical Methods in Natural Language Processing (EMNLP). pages 257-264.

Davide Buscaldi and Paolo Rosso. 2006. Mining knowledge from Wikipedia for the question answering task. In International Conference on Language Resources and Evaluation (LREC). pages 727-730.

Rich Caruana. 1998. Multitask learning. In Learning to learn, Springer, pages 95-133.

Danqi Chen, Jason Bolton, and Christopher D Manning. 2016. A thorough examination of the CNN/Daily Mail reading comprehension task. In Association for Computational Linguistics (ACL).

Ronan Collobert and Jason Weston. 2008. A unified architecture for natural language processing: deep neural networks with multitask learning. In International Conference on Machine Learning (ICML).

Anthony Fader, Luke Zettlemoyer, and Oren Etzioni. 2014. Open question answering over curated and extracted knowledge bases. In ACM SIGKDD international conference on Knowledge discovery and data mining. pages 1156-1165.

David Ferrucci, Eric Brown, Jennifer Chu-Carroll, James Fan, David Gondek, Aditya A Kalyanpur, Adam Lally, J William Murdock, Eric Nyberg, John Prager, et al. 2010. Building Watson: An overview of the DeepQA project. AI magazine 31(3):59-79. 
Clinton Gormley and Zachary Tong. 2015. Elasticsearch: The Definitive Guide. "O'Reilly Media, Inc.”.

Alex Graves, Greg Wayne, and Ivo Danihelka. 2014. Neural turing machines. arXiv preprint arXiv:1410.5401 .

Karl Moritz Hermann, Tomáš Kočiský, Edward Grefenstette, Lasse Espeholt, Will Kay, Mustafa Suleyman, and Phil Blunsom. 2015. Teaching machines to read and comprehend. In Advances in Neural Information Processing Systems (NIPS).

Daniel Hewlett, Alexandre Lacoste, Llion Jones, Illia Polosukhin, Andrew Fandrianto, Jay Han, Matthew Kelcey, and David Berthelot. 2016. Wikireading: A novel large-scale language understanding task over wikipedia. In Association for Computational Linguistics (ACL). pages 1535-1545.

Felix Hill, Antoine Bordes, Sumit Chopra, and Jason Weston. 2016. The Goldilocks Principle: Reading children's books with explicit memory representations. In International Conference on Learning Representations (ICLR).

Minyoung Huh, Pulkit Agrawal, and Alexei A Efros. 2016. What makes ImageNet good for transfer learning? arXiv preprint arXiv:1608.08614 .

Mohit Iyyer, Jordan L Boyd-Graber, Leonardo Max Batista Claudino, Richard Socher, and Hal Daumé III. 2014. A neural network for factoid question answering over paragraphs. In Empirical Methods in Natural Language Processing (EMNLP). pages 633-644.

Rudolf Kadlec, Ondrej Bajgar, and Jan Kleindienst. 2016. From particular to general: A preliminary case study of transfer learning in reading comprehension. Machine Intelligence Workshop, NIPS .

Diederik Kingma and Jimmy Ba. 2014. Adam: A method for stochastic optimization. arXiv preprint arXiv:1412.6980 .

Kenton Lee, Tom Kwiatkowski, Ankur Parikh, and Dipanjan Das. 2016. Learning recurrent span representations for extractive question answering. arXiv preprint arXiv:1611.01436 .

Christopher D Manning, Mihai Surdeanu, John Bauer, Jenny Finkel, Steven J Bethard, and David McClosky. 2014. The stanford corenlp natural language processing toolkit. In Association for Computational Linguistics (ACL). pages 55-60.

Alexander H. Miller, Adam Fisch, Jesse Dodge, AmirHossein Karimi, Antoine Bordes, and Jason Weston. 2016. Key-value memory networks for directly reading documents. In Empirical Methods in Natural Language Processing (EMNLP). pages 14001409.
Mike Mintz, Steven Bills, Rion Snow, and Daniel Jurafsky. 2009. Distant supervision for relation extraction without labeled data. In Association for Computational Linguistics and International Joint Conference on Natural Language Processing (ACL/IJCNLP). pages 1003-1011.

Jeffrey Pennington, Richard Socher, and Christopher Manning. 2014. Glove: Global vectors for word representation. In Empirical Methods in Natural Language Processing (EMNLP). pages 1532-1543.

Pranav Rajpurkar, Jian Zhang, Konstantin Lopyrev, and Percy Liang. 2016. SQuAD: 100,000+ questions for machine comprehension of text. In Empirical Methods in Natural Language Processing (EMNLP).

Pum-Mo Ryu, Myung-Gil Jang, and Hyun-Ki Kim. 2014. Open domain question answering using Wikipedia-based knowledge model. Information Processing \& Management 50(5):683-692.

Minjoon Seo, Aniruddha Kembhavi, Ali Farhadi, and Hannaneh Hajishirzi. 2016. Bidirectional attention flow for machine comprehension. arXiv preprint arXiv:1611.01603.

Huan Sun, Hao Ma, Wen-tau Yih, Chen-Tse Tsai, Jingjing Liu, and Ming-Wei Chang. 2015. Open domain question answering via semantic enrichment. In Proceedings of the 24th International Conference on World Wide Web. ACM, pages 1045-1055.

Zhiguo Wang, Haitao Mi, Wael Hamza, and Radu Florian. 2016. Multi-perspective context matching for machine comprehension. arXiv preprint arXiv:1612.04211.

Kilian Weinberger, Anirban Dasgupta, John Langford, Alex Smola, and Josh Attenberg. 2009. Feature hashing for large scale multitask learning. In International Conference on Machine Learning (ICML). pages $1113-1120$

Jason Weston, Sumit Chopra, and Antoine Bordes. 2015. Memory networks. In International Conference on Learning Representations (ICLR).

Caiming Xiong, Victor Zhong, and Richard Socher. 2016. Dynamic coattention networks for question answering. arXiv preprint arXiv:1611.01604 . 